\title{
La Biblioteca italiana de Haym: le guide d'acquisition de Montesquieu en Italie
}

\section{Eleonora Barria}

\section{Q OpenEdition}

1 Journals

\section{Édition électronique}

URL : http://journals.openedition.org/studifrancesi/5831

DOI : ERREUR PDO dans /localdata/www-bin/Core/Core/Db/Db.class.php L.34 : SQLSTATE[HY000]

[2006] MySQL server has gone away

ISSN : 2427-5856

\section{Éditeur}

Rosenberg \& Sellier

Édition imprimée

Date de publication : 1 mai 2011

Pagination : 80-85

ISSN : 0039-2944

\section{Référence électronique}

Eleonora Barria, « La Biblioteca italiana de Haym: le guide d'acquisition de Montesquieu en Italie », Studi Francesi [En ligne], 163 (LV | I) | 2011, mis en ligne le 30 novembre 2015, consulté le 08 janvier 2021. URL : http://journals.openedition.org/studifrancesi/5831; DOI : https://doi.org/ERREUR PDO dans /localdata/www-bin/Core/Core/Db/Db.class.php L.34 : SQLSTATE[HY000] [2006] MySQL server has gone away

\section{(c) (i) (9)}

Studi Francesi è distribuita con Licenza Creative Commons Attribuzione - Non commerciale - Non opere derivate 4.0 Internazionale. 


\section{La Biblioteca italiana de Haym: le guide d'acquisition de Montesquieu en Italie}

Ayant consacré notre thèse de doctorat à l'analyse la «bibliothèque italienne» de Montesquieu ${ }^{1}$, nous avons choisi de commenter cette collection à la lumière d'un seul ouvrage: la Biblioteca italiana o Notizia de' libri rari nella lingua italiana $[. . .]^{2}$ de Francesco Nicola Haym³. Probablement acheté à Venise par Montesquieu, la Biblioteca italiana de Haym est consignée dans le Catalogue de La Brède ${ }^{4}$. Comme son titre l'indique, cet ouvrage est une «bibliothèque», terme désignant au XVIII siècle les répertoires bibliographiques universels ou spécialisés ${ }^{5}$. Or, le Catalogue de Montesquieu présente une division intitulée «De Bibliothecis, librorum indicibus et catalogis scriptores», où sont rassemblées les rubriques mentionnant les «bibliothèques», les «catalogues» ${ }^{6}$ et les «index» des livres, c'est-à-dire des recueils de titres, souvent organisés par disciplines ou sujets, qui aident les collectionneurs dans leur recherche. Mais, si l'un des plus importants collectionneurs du XVIII siècle, Pierre Amadoli', classe parmi ses «bibliothèques» la Biblioteca italiana de Haym, seul «usuel» éclairant les bibliophiles sur la production littéraire italienne ${ }^{8}$, Montesquieu fait insérer le volume de Haym dans la division «Literatores, philologi, oratores, critici, grammatici, etc» de son Catalogue. Ce choix nous laisse deviner que pour Montesquieu la Biblio-

(1) Traiter de la «bibliothèque italienne» de Montesquieu signifie rendre compte des livres réunis dans le Catalogue de la bibliothèque de Montesquieu à La Brède, notamment des ouvrages d'auteurs italiens en latin, en italien ou en traduction et d'auteurs français ou étrangers qui ont écrit en français sur l'Italie. À ces ouvrages s'ajoutent les quelques textes de la bibliothèque parisienne de Montesquieu mentionnés dans la dernière édition du Catalogue, les livres à propos desquels nous renseignent les notes des libraires, les extraits de lectures et quelques fragments consignés dans les Pensées, le Spicilège et la Correspondance, de même que les études qui ont tenté de reconstruire l'univers livresque de Montesquieu.

(2) N. F. Haym, Biblioteca italiana osia Notizia de' libri rari nella lingua italiana [...] Annessovi tutto il libro della Eloquenza italiana di Mons. Giusto Fontanini, Venezia, A. Geremia, 1728. Par la suite, nous ferons toujours référence à cette édition grâce à l'abréviation: HAYM (1728).

(3) Francesco Nicola Haym naît à Rome le 6 juillet 1678 et meurt à Londres le 31 juillet 1729 . Avant son décès et en parallèle avec son activité de musicien, Haym est célèbre pour d'autres activités: les ouvrages littéraires, le collectionnisme et la recherche antiquaire. Son activité de collectionneur touche à la numismatique, mais aussi à la littérature. C'est pourquoi en 1726 Haym est le compilateur de la Biblioteca Italiana o sia Notizia de' libri rari nella lingua italiana. Cet ouvrage, publié pour la première fois en Italie en 1728 à Venise, chez Angiolo Geremia, sera réimprimé en Italie jusqu'à 1803 . Voir Dizionario biografico degli Italiani, Roma, Isti-tuto dell'Enciclopedia Italiana, depuis 1960, t. 61, pp. 667-669.

(4) Le manuscrit du Catalogue de la Bibliothèque de Montesquieu à La Brède est conservé à Bordeaux (Bibliothèque municipale de Bordeaux: MS 2539, fonds de La Brède), tandis que l'édition plus récente de celui-ci remonte à 1999: L. Desgraves et C. Volpilhac-Auger, Catalogue de la Bibliothèque de Montesquieu à La Brède, avec la collaboration de F. WEIL, «Cahiers Montesquieu», n. 4, Napoli, Liguori, Paris, Universitas, Oxford, Voltaire Foundation, 1999. Par la suite, nous ferons toujours référence à cette édition grâce à l'abréviation: Catalogue. Pour la Biblioteca italiana de Haym voir Catalogue, n. 1828.

(5) Y. SORDET, Une approche des catalogues domestiques de bibliothèques privées (XVIIe-XVIIIe siècles), instruments et miroirs de collections particulières, «Bulletin du bibliophile», 1997, n. 1, p. 93.

(6) Catalogue, nn. 2542-2557.

(7) Pierre Amadoli (1707-1769). Pour tout renseignement bio-bibliographique sur ce personnage voir Y. SORDET, L'Amour des livres au siècle des Lumières. Pierre Amadoli et ses collections, préface de D. Roche, Paris, École des Chartes, 2001.

(8) Ibid., p. 204 et p. 234. 
teca italiana n'est pas seulement un répertoire bibliographique, mais aussi un ouvrage destiné aux hommes de lettres et, par conséquent, un instrument témoignant de la façon dont Montesquieu conçoit les Belles-Lettres italiennes.

C'est pourquoi, il est nécessaire de mettre en avant la singularité de ce texte qui a fort probablement rempli la fonction de source bibliographique de Montesquieu, acheteur de livres italiens pendant son voyage en Italie, du 16 août 1728 au 29 juillet 1729. De même, nous verrons dans quelle mesure la Biblioteca italiana de Haym est un outil nécessaire à la caractérisation du fonds en langue italienne de La Brède. La Biblioteca italiana de Haym se présente comme un vrai libro italico, étant donné que l'auteur, le lieu d'édition et la langue employée sont italiens. En effet, ce texte ne mentionne aucun auteur étranger ayant écrit à propos de l'Italie et il ne cite qu'exceptionnellement les impressions étrangères et presque jamais les traductions. Cet ouvrage offre donc une sélection d'auteurs et de titres italiens, répartis selon un classement systématique en quatre parties, consacrées à l'histoire, aux poètes, aux livres en prose, aux arts et aux sciences et comprenant plusieurs sous-catégories. Cette organisation et la présence de la table alphabétique de toutes les histoires, des noms et des prénoms des auteurs simplifient la consultation de ce livre que Haym définit comme un simple catalogue d'auteurs italiens? .

Comme le souligne l'éditeur de l'impression de 1803, Giovanni Silvestri ${ }^{10}$, au cours du XVIII ${ }^{\mathrm{e}}$ siècle ce catalogue devient indispensable pour ceux qui désirent connaître les œuvres italiennes remarquables pour le mérite de l'auteur et du sujet, mais aussi pour la rareté ou la qualité de l'impression. C'est pourquoi, chaque notice bibliographique est accompagnée d'un commentaire portant sur l'auteur, sur la valeur historique, littéraire ou philologique de l'ouvrage, mais aussi sur les caractéristiques bibliographiques et matérielles de l'impression. Enfin, un astérisque met en évidence les livres les plus rares de sa sélection ${ }^{11}$ et trois termes «Raro, molto Raro, Rarissimo» ${ }^{12}$ permettent une déclinaison de leur rareté.

La notion de rareté, catégorie fondamentale pour l'évaluation et l'insertion d'un livre dans ce catalogue, est un élément essentiel de la description des ouvrages. Or, cette notion mérite d'être éclairée, étant donné que quelques indices nous encouragent à croire que pour Haym, comme pour ses lecteurs, la «rareté» est à la fois un critère bibliophilique et esthétique. Dans l'édition de 1691 du Vocabolario della Crusca, répertoriée par Haym, la rareté, «rarità», est le nom abstrait créé à partir de l'adjectif «raro», signifiant «rado» et donc rare, c'est à dire le contraire de fréquent, d'épais, de dense $^{13}$. Désignant ce qui se rencontre peu souvent ou dont il existe peu d'exemplaires, le terme «rari», qualifiant les livres réunis dans la Biblioteca italiana, caractérise des ouvrages qui ont d'autant plus de valeur qu'ils sont peu répandus. Précieux par leur rareté, ces volumes le sont aussi en raison de leur excellence.

Si la rareté, qui intéresse tant les bibliophiles, est mentionnée dans le titre choisi par Haym, l'excellence des volumes est le critère esthétique respecté par Giusto Fontanini lors de la constitution de son «Catalogo delle opere più eccellenti che intorno alle principali arti, e facoltà sono state scritte in lingua italiana» ${ }^{14}$, figurant

(9) Dans l'épître dédicatoire adressée par Haym à son Excellence Mylord Comte de Winchilsea, la Biblioteca italiana est définie comme un «semplice $\mathrm{Ca}$ talogo di Autori Italiani». Voir «All'Eccellenza di Mylord Conte di Winchilsea», HaYM (1728), non paginé.

(10) «L'Editore a chi legge», N. F. HAYM, Biblioteca italiana ossia Notizia de' libri rari nella lingua italiana..., Milano, Presso Giovanni Silvestri, 1803, 4 vol., non paginé. Par la suite nous ferons tou- jours référence à cette édition grâce à l'abréviation: HAYM (1803).

(11) Voir «A' Lettori», HAYM (1728), non paginé.

(12) [Rare, très rare, rarissime].

(13) Vocabolario della Crusca (1691), t. III. Articles: «Raro», p. 1324 et «Rado», p. 1309.

(14) [Catalogue des œuvres les plus excellentes qui ont été écrites en langue italienne sur les arts et 
dans Dell'Eloquenza italiana et intégralement reproduit dans la Biblioteca italiana de Haym. Lorsqu'il intitule son ouvrage Dell'Eloquenza italiana Fontanini suit l'exemple du De Vulgari eloquentia de Dante et utilise le terme «eloquenza», comme synonyme de «favella», à savoir de langue ${ }^{15}$. Ce faisant, il se propose de traiter de l'évolution de la langue italienne et des écrits produits dans cet idiome, pour prouver que, contrairement aux affirmations du Père Bouhours parues dans De la Manière de bien penser de 1687 , l'ancienne valeur des lettres italiennes existe toujours ${ }^{16}$. C'est pourquoi, dans Dell'Eloquenza italiana, Fontanini souligne la dignité de la langue italienne qui, ancienne de quatre siècles, n'est pas jugée à sa juste valeur par les lecteurs français, dont la langue est jeune de cinquante ans ${ }^{17}$; puis, il conclut son ouvrage dressant le catalogue des «plus excellentes» ouvres italiennes, également appreciées par les lecteurs italiens et étrangers ${ }^{18}$.

Utilisant le superlatif «eccellenti» ${ }^{19}$, Fontanini affirme la perfection esthéticolittéraire atteinte à son avis par tous les ouvrages qu'il sélectionne et qu'il considère comme les meilleurs. Par son classement, Fontanini exprime un jugement de valeur et produit un répertoire d'auteurs italiens établi grâce aux critères esthétiques de l'excellence et de la perfection littéraires, auxquels semble adhérer Haym lorsqu'il choisit d'insérer Dell'Eloquenza italiana dans la Biblioteca italiana. Du fait que dans son catalogue Haym publie et enrichit la liste des livres répertoriés par Fontanini et qu'il reproduit le «Ragionamento Dell'Eloquenza italiana», à savoir la réflexion sur l'éloquence italienne que Fontanini adresse au marquis bolonais Orsi, le 30 juin 1706, Haym se range du côté des principaux savants italiens du XVIII ${ }^{e}$ siècle, tels que le marquis Orsi, Scipione Maffei et Ludovico Muratori, dans la défense de la production italienne. En effet, reproduisant Dell'Eloquenza italiana et le «Ragionamento Dell'Eloquenza italiana» de Fontanini dans la Biblioteca italiana, Haym semble adhérer au projet de son prédécesseur et s'inscrit dans la querelle Orsi-Bouhours ${ }^{20}$. C'est sûrement en raison de cela que Montesquieu décide de classer la Biblioteca italiana dans la division «Literatores, philologi, oratores, critici, grammatici, etc» et non pas dans la division «De Bibliothecis, librorum indicibus et catalogis...» du Catalogue.

Ainsi, la Biblioteca italiana de Haym rassemble toutes les meilleures œuvres en langue italienne: les éditions les plus rares, à savoir les moins répandues et les plus précieuses pour les bibliophiles, et les impressions qui, selon une certaine esthétique littéraire, sont considérées comme les plus remarquables. Par son contenu et ses critères de classement la Biblioteca italiana intéresse donc les amateurs et les connaisseurs des lettres italiennes, mais aussi les collectionneurs et les bibliographes du XVIII ${ }^{\mathrm{e}}$ siècle.

Catalogue destiné aux érudits qui peuvent l'apprécier en tant qu'ouvrage conçu comme un outil instructif et nécessaire ${ }^{21}$, rédigé par Haym à la suite de la consultation de nombreuses sources d'autoritée $e^{22}$ la Biblioteca italiana est aussi un instrument au

les facultés principaux]. Voir G. Fontanini, Della Eloquenza italiana [...], Roma, per Francesco Gonzaga, 1706.

(15) «Ragionamento di Mons. Giusto Fontanini Della Eloquenza italiana all'Illustriss. Sig. Marchese Gian Giuseppe Orsi», Haym (1728), non paginé.

(16) Ibid., (art. IV).

(17) Ibid., (art. XII).

(18) Ibid.

(19) Vocabolario della Crusca (1691), t. II, p. 587 .

(20) A propos de la querelle Orsi-Bouhours voir C. VIOLA, Tradizioni letterarie a confronto. Italia e Francia nella polemica Orsi-Boubours, Verona, edi- zioni Fiorini, 2001.

(21) «A' Lettori», HaYm (1728), non paginé.

(22) Ce collectionneur privé consulte les plus importantes bibliothèques privées et publiques, les catalogues et les répertoires bibliographiques imprimés à l'époque, tandis qu'il exclut de ses sources les catalogues de vente des libraires, car, souvent rédigés par ces derniers ou par des gens peu intelligents, ils sont selon Haym peu exacts. L'exactitude et la qualité de la sélection sont donc pour Haym les critères indispensables à la compilation de son catalogue qu'il se propose d'enrichir davantage grâce à la consultation des catalogues de la «Biblioteca Napoletana e Fiorentina». Voir ibid. 
service des «letterati». C'est pourquoi, comme l'indique l'éditeur milanais Giovanni Silvestri en 1803, au cours du XVIII siècle, ce volume, consacré aux livres italiens rares, devient lui-même rarissime et onéreux ${ }^{23}$, car les nombreuses réimpressions ne suffisent pas à satisfaire la demande de son principal public, à savoir les collectionneurs, les bibliographes et les bibliophiles italiens et étrangers comme Pierre Amadoli.

Texte compilé par un collectionneur privé, qui dévient l'un des plus célèbres bibliographes de tout le XVIII ${ }^{\mathrm{e}}$ siècle ${ }^{24}$, la Biblioteca italiana s'impose donc en tant que référence officielle des amateurs et des connaisseurs des lettres italiennes, de même qu'il figure parmi les «usuels» des érudits et des bibliophiles européens du XVIII siècle. C'est pourquoi, la singularité de ce texte et sa diffusion auprès de ce dernier type de lecteurs nous encouragent à justifier sa présence dans le fonds italien de la bibliothèque de Montesquieu à La Brède.

Du fait que la circulation des livres italiens en France au XVIII ${ }^{\mathrm{e}}$ siècle est très désavantagée et que les ouvrages contemporains arrivent même avec plusieurs années de retard à Paris ${ }^{25}$, Montesquieu a sûrement profité de son séjour en Italie pour se procurer la Biblioteca italiana, qui, impression vénitienne de 1728, est fort probablement l'une des acquisitions réalisées par le Président pendant son séjour dans la Sérénissime, du 16 août au 15 septembre 1728. Or, étant donné la nature spécifique de ce texte, nous avons vérifié la présence des livres du fonds en langue italienne de La Brède dans la Biblioteca italiana. Or, quarante-deux des soixante-dix-huit ouvrages en italien présents à La Brède sont mentionnés par Haym. Le fonds en langue italienne de Montesquieu regroupe donc plusieurs ouvrages rares et bien vingt-trois ouvrages très rares, selon les critères de Haym. Ce constat permet de formuler deux hypothèses. Comme la Biblioteca italiana de Haym est un «usuel» aidant les collectionneurs et les bibliophiles du XVIII siècle dans la recherche des livres rares, Montesquieu pourrait avoir employé ce volume pour vérifier le contenu du fonds italien de sa bibliothèque de La Brède, dont il hérite en 1716. Mais, étant donné que le nombre des ouvrages du fonds italien de La Brède figurant dans l'ouvrage de Haym dépasse considérablement celui des libri italici rares conservés dans les catalogues bordelais que nous avons étudiés, notamment ceux des Présidents et bibliophiles Arnaud de Pontac et Barbot, Montesquieu pourrait aussi avoir utilisé ce catalogue comme source bibliographique. La Biblioteca italiana pourrait donc être à l'origine des acquisitions italiennes remontant au séjour italien de Montesquieu et avoir inspiré les projets d'achat enregistrés dans le fragment n. 660 du Spicilège:

Acheter à Naples le Vasari sur la peinture non impression de Bologne qui est en deux vol mais de Florence en trois volumes

carte du Pô

Giannone Histoire de Naples [...]

Les livres sur peinture: Leonard de Vinci [,] Vasari [,] Junius victura veterum [,]Vignole sur l'architecture

Sur la perspective

Perspectiva, di Vignola commentata dal padre Danti

Perspectiva del Accolti inganno dell'occhio

Perspectiva del cavalier Sirigati

Perspectiva del pade Pozzi

Perspectiva del Troili vaut six paules

(23) «L'Editore a chi legge», HAYM (1803), non paginé.

(24) Ibid.
(25) F. Waquet, Le Modèle français et l'Italie savante (1660-1750), Rome, École Française de Rome, 1989, pp. 31-41. 
Architecture

Edition du Palladio celle d'Angleterre la meilleure et elle coute beaucoup: celle de Venise une pistole:

Scamozi la première edition de Venise 3 pistoles le 2de une pistole mais mauvaise

Le Serlio en sept livres l'in-folio est le meilleur en un thome; l'in-quarto, un thome seul plus facile a trouver ${ }^{26}$.

Bien que ce fragment autographe paraisse parmi ceux recopiés par le secrétaire $e$, entre 1736-1737 27 , plusieurs indices nous encourageraient à le considérer comme une liste d'ouvrages contemporaine du voyage de Montesquieu en Italie et remaniée après son retour en France. Or, ces ouvrages, qu'il aurait voulu acheter à Naples, n'ont jamais été acquis par Montesquieu, mais, témoignant de son intérêt pour les lettres italiennes, ils ont été identifiés par Rolando Minuti et Salvatore Rotta ${ }^{28}$. Compte tenu des observations que Montesquieu consacre à la qualité, au prix, au format et à l'impression des ouvrages cités dans ce fragment, nous avons comparé les éditions identifiées avec celles répertoriées dans la Biblioteca italiana de Haym: l'Historia de Giannone, mais aussi la plupart des ouvrages des artistes italiens sur l'architecture et la peinture sont mentionnés par Haym parmi les livres rares et même très rares.

Ainsi, la présence chez Haym de quarante-deux des soixante-dix-huit ouvrages en italien présents à La Brède et de neuf des quatorze ouvrages cités dans le fragment n. 660 du Spicilège nous encourage à croire que Montesquieu se sert de la Biblioteca italiana de Haym comme source et guide bibliographiques pour ses acquisitions contemporaines du voyage et pour ses projets d'achat. Par ailleurs, les remarques sur les impressions réunies dans le fragment n. 660 témoignent d'un lecteur averti et exigeant qui choisit attentivement les ouvrages à acquérir et prévoit l'achat de livres que l'auteur, le sujet traité, mais aussi la qualité de l'impression rendent tout à fait remarquables, rares ou même très rares, selon les critères de Haym.

Enfin, l'existence dans le fonds italien de la bibliothèque de Montesquieu à La Brède de quarante-deux «livres rares» permet de revoir les jugements exprimés par Gustave Brunet sur la bibliothèque de Montesquieu dans ses Fantaisies bibliographiques $^{29}$ de 1864 sûrement à la lumière de l'inventaire rédigé en 1756, à la demande de Jean-Baptiste de Secondat ${ }^{30}$. Brunet affirme que la «condition des livres qui appartenaient à l'illustre publiciste est des plus ordinaires» ${ }^{31}$ et, s'intéressant à la valeur marchande de cette collection, il précise que «la bibliothèque de Montesquieu offre tout ce que l'antiquité a produit de plus important. Satisfait d'avoir les textes, le propriétaire ne s'est point attaché à réunir les éditions les plus estimées pour la critique et pour les travaux des commentateurs. Rien n'a été donné au luxe; tous les volumes rassemblés à La Brède sont des livres de travail, et beaucoup d'entre eux portent les traces du long usage qui en a été fait» ${ }^{32}$.

Puis, Brunet commente brièvement la présence à La Brède de quelques ouvrages italiens: il cite le voyage de «Marco-Polo, en italien, édition de 1559» ${ }^{33}$, dans la section «Histoire», et celui de «Falda, sur les fontaines de Rome» ${ }^{34}$, dans la division «Scien-

(26) Montesquieu demeure en Italie du 16 août 1728 au 29 juillet 1729. Pour le fragment n. $660 \mathrm{du}$ Spicilège: voir Montesquieu, Spicilège, Euvres complètes, édition R. Minuti et S. RotTA, Oxford, Voltaire Foundation, 2002, t. 13 , p. 579.

(27) Il s'agit des fragments nn. 656 et 663 . Voir ibid., pp. 573 et 581. Le secrétaire $e$ est au service de Montesquieu de 1738 à 1739. Sur les secrétaires de Montesquieu qui recopient les fragments du Spicilège, voir «Introduction», ibid., pp. 50-62.
(28) Voir ibid., pp. 580-581.

(29) Paris, 1864, p. 138-144. Voir L. Desgraves, «Introduction», Catalogue, p. 9.

(30) Ibid.

(31) Ibid., p. 10.

(32) Ibid., p. 11.

(33) Ibid. Cet ouvrage ne figure pas dans le $\mathrm{Ca}$ talogue.

(34) Ibid., p. 10. 
ces et les Arts». Évoquant le seul voyage de Marco-Polo, Brunet semble ignorer les descriptions historiques, artistiques et géographiques des villes italiennes qui s'inscrivent dans le genre de la littérature de voyage, de même que les ouvrages de plusieurs historiographes remarquables, tels que Bosio, Bentivoglio, Davila, Giovio, Nani et Siri. Ne mentionnant que l'ouvrage de Falda, il passe sous silence les ouvrages de Malvasia sur les peintres bolonais et les volumes consacrés aux peintures de Raphaël et à l'architecture de Vitruve. Par ailleurs, aucune référence n'est faite aux livres sur l'art de fortifier et de combattre, à l'ouvrage mathématique de Galilée et au volume philosophique d'Algarotti. Enfin, au moment d'analyser la collection des «Belles-Lettres», Brunet affirme que «la part de la littérature italienne est assez faible» ${ }^{35}$, ce qui est assez étonnant car les Belles-Lettres sont la catégorie bibliographique du fonds en langue italienne la mieux pourvue d'impressions. Il termine en précisant que «Pétrarque ne se montre que dans une édition toute récente de 1711; 1'Arioste est de 1566; la Jérusalem délivrée de 1581 est une des plus anciennes éditions» ${ }^{36}$.

Brunet qualifie donc la bibliothèque de Montesquieu de bibliothèque de travail, sans reconnaître la valeur et la rareté de beaucoup d'éditions italiennes anciennes des $\mathrm{XVI}^{\mathrm{e}}$ et XVIII ${ }^{\mathrm{e}}$ siècles, ni la qualité de certaines éditions plus récentes, comme celle des Rimes de Pétrarque. En effet, cette édition, annotée par Alessandro Tassoni et Ludovico Antonio Muratori est mentionnée par Montesquieu dans le Voyage en Italie ${ }^{37}$ et figure parmi les livres rares cités par Haym, qui précise que cet ouvrage est généra-

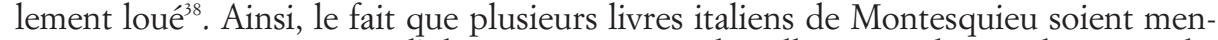
tionnés par Haym permet d'observer autrement la collection italienne de La Brède. En effet, bien que le fonds en langue italienne soit caractérisé par un nombre limité d'ouvrages, il a néanmoins le mérite et la particularité de réunir dans cette bibliothèque privée de province des œuvres que l'un des plus célèbres bibliographes du XVIII ${ }^{\mathrm{e}}$ siècle juge de grande qualité littéraire et considère comme rares, parfois rarissimes, dignes d'un grand connaisseur de la production italienne ou d'un vrai bibliophile.

Pour conclure, la présence d'un texte aussi singulier que la Biblioteca italiana de Haym dans la bibliothèque de Montesquieu à La Brède et le fait que quarante-deux des soixante-dix-huit ouvrages en italien de Montesquieu y paraissent nous permettent de revoir le jugement porté par Brunet sur le fonds en langue italienne et sur Montesquieu collectionneur. En effet, la présence dans le fonds en langue italienne d'autant d'ouvrages rares, ainsi que les précisions de Montesquieu sur les ouvrages italiens très précieux qu'il veut acheter et qu'il enregistre dans le fragment n. 660 du Spicilège, témoignent d'un lecteur qui prévoit l'achat de livres qu'il choisit en fonction de la qualité de leur contenu et de leur impression. Ainsi, digne élève du collège de Juilly respectueux du sixième entretien du Père Lamy, prônant la lecture des meilleurs livres dans les meilleures éditions ${ }^{39}$, Montesquieu est probablement un lecteur attentif aux qualités littéraires et bibliophiliques des ouvrages. C'est pourquoi il a sans doute utilisé la Biblioteca italiana de Haym en tant que source bibliographique et guide pour ses acquisitions et ses projets d'achat datant de son voyage en Italie et peut-être aussi pour vérifier, dès son retour en France, la qualité des ouvrages hérités de ses ancêtres en 1716.

ELEONORA BARRIA

(35) Ibid.

(36) Ibid.

(37) Montesquieu, Voyage en Italie, Euvres complètes, édition A. MAsson, Paris, Nagel, 19501955, 3 vol., t. II, p. 1216.

(38) HAYM (1728), p. 104.2

(39) Voir, L. DeSGRAVES, «Introduction», Montesquieu, Pensées, Le Spicilège, édition L. DesGra-
VES, Paris, Robert Laffont, 1991, coll. «Bouquins», p. 10. Montesquieu possède l'édition lyonnaise des Entretiens sur les Sciences du Père Lamy, datée de 1694 (voir Catalogue, n. 1449): «on y apprend comme l'on doit se servir des sciences pour se faire l'esprit juste et le cœur droit et acquérir une méthode de travail». 